\title{
Estudantes de Medicina e as Drogas: Evidências de um Grave Problema
}

\section{Medical Students and Drugs: Evidences of a Serious Problem}

\author{
Cleomara de Souza Machado \\ Talles Mendes de Moural \\ Rogério José de Almeida
}

\section{PALAVRAS-CHAVE \\ - Drogas; \\ - Transtornos Relacionados ao Uso de Substâncias; \\ - Estudantes de Medicina; \\ - Prevalência; \\ - Educação Médica.}

\section{KEYWORDS}

- Drugs;

- Substance-Related Disorders;

- Medical Students;

- Prevalence;

- Medical Education.
Recebido em:05/06/2014

Aprovado em: 17/10/2014

\section{RESUMO}

Este artigo analisa a prevalência e os fatores que desencadeiam o consumo de drogas entre estudantes de Medicina. Trata-se de uma revisão integrativa da literatura científica acerca da prevalência do uso de drogas em estudantes de Medicina.Optou-se por privilegiar periódicos de indexação científica, consultando-se o Pubmed e a Biblioteca Virtual em Saúde, e utilizando-se as bases de dados SciELO,Medline e Lilacs. São analisadas três categorias do fenômeno: prevalência do uso de drogas entre os estudantes de Medicina (categoria dividida em drogas lícitas e ilícitas); fatores que propiciam o uso de drogas entre os estudantes de Medicina; e análise referente aos estudantes brasileiros de Medicina. Constatam-se evidências de um grave problema nas Faculdades de Medicina, que é a grande e constante prevalência do uso de drogas lícitas ou ilícitas entre os estudantes e fatores intrínsecos ao curso que podem desencadear o início ou a continuidade dessa prática. Esse problema requer a atenção dos diversos representantes das universidades a fim de que se adotem políticas de controle e redução de uso de drogas no âmbito universitário.

\begin{abstract}
This article aims to analyze the prevalence of, and the factors that trigger drug use among medical students. This is an integrative review of the scientific literature on the prevalence of drug use in medical students. We chose to focus on indexed scientific journals, querying Pubmed and the Virtual Health Library, using the SciELO, Medline and Lilacs databases. Three categories of the phenomenon are analyzed: prevalence of drug use among medical students (divided into categories of licit and illicit drugs); factors that promote drug use among medical students; and an analysis specifically in relation to Brazilian medical students. Evidence was found of a serious ongoing problem in medical schools; the major and constant prevalence of licit and illicit drug use among their students and intrinsic factors of the courses that may be trigger or serve to sustain such behavior. This problem deserves the attention of various university representatives to implement policies to control and reduce drug use in the university setting.
\end{abstract}




\section{INTRODUÇÃO}

O uso e abuso de drogas lícitas e ilícitas, também chamadas substâncias psicoativas, é uma prática corrente desde os primórdios da humanidade. A partir do início do século $X X$, dentre os problemas enfrentados pelos estados modernos, o crescimento da circulação e uso de substâncias psicoativas ganha destaque. Nesta perspectiva histórica, pode-se dizer que o uso de drogas se tornou um problema de saúde pública global em razão da dependência gerada, dos efeitos produzidos pela progressão no consumo, do poli uso, do abuso crônico e da gravidade de suas consequências. É um grave problema, que ultrapassa o limite biológico, culminando em um aumento vertiginoso da prevalência do consumo na população mundial ${ }^{1}$.

No Brasil,em meados do século XX, houve um crescimento significativo do consumo de drogas para fins recreativos, modificação do humor, busca de prazer, alívio de ansiedade e estresse cotidiano, em idades cada vez mais precoces.Santos e Oliveira ${ }^{1}$ afirmam que a substância psicoativa é transformada em "droga" em virtude da simbolização que assume para determinados sujeitos ou grupos sociais.

Sendo assim, entende-se por substâncias psicoativas aquelas que, quando ingeridas ou administradas no organismo, são capazes de alterar os processos mentais e cognitivos dos indivíduos. Este termo e seu equivalente, droga psicotrópica, são os termos mais neutros e descritivos para toda a classe de substâncias lícitas (ex. álcool) e ilícitas (ex. maconha, cocaína). O termo "psicoativas" não implica necessariamente a produção de dependência. Em linguagem comum, muitas vezes,ele é deixado implícito, como em "uso de drogas" ou "abuso de substâncias"2.

Entre as drogas de uso prevalente na população encontram-se o álcool e o tabaco. São substâncias que merecem uma diferenciação, pois são consideradas drogas lícitas, ou seja, são livremente comercializadas e, sobretudo, socialmente aceitas, mas que nem por isso deixam de causar os mesmos males à saúde acarretados pelas drogas ilícitas, cuja produção, venda e uso são legalmente proibidos no Brasil ${ }^{3}$.

O excesso de consumo de álcool tem sido citado como um dos principais custos evitáveis para a sociedade, pelas enfermidades que causa. Tem sido associado a muitos ferimentos evitáveis, tais como acidentes automobilísticos, quedas, afogamentos, homicídios, suicídios, abuso infantil e violência doméstica, bem como a numerosas consequências para a saúde, como intoxicação alcoólica, hipertensão, ataques cardíacos, pancreatite e cirrose hepática ${ }^{4}$.

Os jovens, não somente brasileiros,mas também de outras nacionalidades, vêm cultivando o hábito de se embriagar. No
Brasil, a prevalência do uso de drogas no ensino superior é um problema que precisa ser enfrentado. $\mathrm{O}$ ambiente universitário parece facilitar o acesso dos jovens às drogas e identificou-se também que o consumo aumenta após a inserção na faculdade $^{5}$. É importante destacar que há maior prevalência do consumo de álcool, tabaco e outras drogas entre universitários brasileiros do que na população em geral' .

Com relação aos universitários do curso de Medicina, as pesquisas têm demonstrado índices elevados de consumo de drogas, que constitui uma das inúmeras "válvulas de escape" para os problemas psicológicos ou de resiliência provocados pela rotina estressante. $\mathrm{O}$ álcool é a substância mais utilizada no cotidiano desses estudantes, com percentuais de até $98 \%$, seguido por tabaco, maconha, solventes e tranquilizantes. Há relatos de que o consumo de álcool e outras drogas está presente de forma "endêmica" na comunidade médica, e, frequentemente, seu uso se inicia durante a graduação $\mathrm{o}^{7,8}$.

Com base em vivências pessoais no curso de Medicina, seguidas da observação das atitudes dos estudantes de Medicina diante do enfrentamento das dificuldades na formação médica, é possível considerar que inúmeros fatores podem contribuir para o uso indevido de drogas no período da formação superior. Ao ingressar no curso de Medicina, o estudante necessita se adaptar a uma realidade nova de ensino-aprendizagem, de vida universitária, de mudança no estilo de vida e de aquisição de maiores responsabilidades, para as quais talvez ainda não esteja preparado. Surge, assim, um conflito interpessoal, que pode levá-lo a buscar meios para "fugir" dessa realidade.

Parte-se do princípio de que as características do curso de Medicina têm atuado como fatores de risco para que o estudante dê início e/ou continuidade ao uso/abuso de drogas, sejam elas lícitas ou ilícitas. Os alunos, de forma intensiva, são submetidos a carga horária elevada de estudos e a extensos conteúdos durante as aulas, sendo necessária a continuidade dos estudos em casa. Portanto, resta pouco tempo para estar com a família e com os amigos, realizar atividades fora da realidade médica e cuidar da própria saúde ${ }^{9}$.

Os estudantes do curso de Medicina estão constantemente em contato com o processo de saúde, doença e morte, levando consigo um sentimento quase obrigatório de curar o enfermo. A morte de um paciente, para determinados estudantes, leva, por vezes, a um inevitável evento traumático que pode perdurar por toda a sua graduação e comprometer a formação médica. Fatores como sair de sua cidade, morar sozinho, pressão psicológica, abuso de autoridade dos preceptores e métodos institucionais avaliativos colaboram significativamente para a interferência no estado psíquico dos estudantes. Frente a isso, 
não é raro identificar alunos ansiosos pela chegada de um final de semana para extravasar o estresse por meio de drogas, principalmente as bebidas alcoólicas ${ }^{10}$.

Muitos desses jovens têm dificuldades em suportar as situações estressantes na vida acadêmica, não conseguem lidar bem com os processos de adaptação ou até mesmo se recuperarde momentos mais complexos durante a formação. Por isso, estão surgindo informações e reflexões a respeito do cuidado psicológico que se deve ter com os estudantes de Medicina, levando em conta a importância de não se formar um profissional doente.

Emerge, então, uma grande preocupação com os estudantes de Medicina, cuja formação é voltada para a promoção da saúde. Se eles são os doentes, esse objetivo fica prejudicado, e consequências podem surgir durante e após a vida universitária. Em toda a sua formação acadêmica, voltada para o outro como sendo o doente e com necessidade de atenção, nota-se que não é comum que esses estudantes reconheçam os próprios problemas psicossociais. Isto pode levá-los, futuramente, a enfrentar dificuldades quanto aos diagnósticos e aos tipos de tratamento que se devem estabelecer a pacientes com tais queixas.

Pesquisas nacionais e internacionais vêm avaliando as atitudes e comportamentos dos acadêmicos de Medicina frente ao tema drogas. Esses estudos possibilitam o entendimento de comportamentos, assim como o desenvolvimento de estratégias de prevenção do uso e abuso de substâncias psicoativas e, como consequência, a tentativa de melhorar a qualidade de vida desses estudantes ${ }^{11}$.

Daí o nosso interesse em resgatar na literatura científica, por meio de uma revisão integrativa, resultados de pesquisas que procuram responder ao seguinte questionamento: Quais as características da prevalência e os fatores desencadeantes existentes na relação entre estudantes de Medicinas e as drogas?

Portanto, por meio da análise de pesquisas científicas publicadas de 2008 a 2013, o presente estudo examina a prevalência e os fatores que desencadeiam o consumo de drogas entre estudantes de Medicina. Esta é uma pesquisa realizada de maneira sistemática e ordenada com base no referencial teórico da Prática Baseada em Evidências, que busca proporcionar uma síntese de múltiplos estudos publicados que facilita a transposição dessas evidências para a prática clínica.

\section{METODOLOGIA}

Este artigo constitui uma revisão integrativa da literatura científica acerca da prevalência do uso de drogas em estudantes de Medicina. Para o alcance do objetivo proposto, optou-se por este tipo de revisão de literatura, uma vez que ela apresenta a síntese de múltiplos artigos científicos. Além disso, possibilita conclusões gerais de determinada área de estudo, contribuindo para o aprofundamento e difusão do conhecimento do fenômeno investigado e de como ele tem sido analisado nas pesquisas atuais ${ }^{12}$.

A revisão integrativa é um estudo em que se realiza a coleta de dados de fontes secundárias por meio de um rigoroso levantamento bibliográfico que possibilita reunir conhecimentos sobre o fenômeno a ser investigado. Constitui uma técnica de pesquisa com rigor metodológico, criteriosa e conscienciosa, que aumenta a confiabilidade e a profundidade das conclusões $^{12}$.

Esse tipo de revisão é muito difundido entre as abordagens relacionadas à Prática Baseada em Evidências, que é amplamente aceita no âmbito dos estudos que visam a uma análise de solução de problemas para a tomada de decisão que incorpora a busca da melhor e mais recente evidência. Envolve a definição de um problema, a busca e a avaliação crítica das evidências disponíveis, a implementação das evidências na prática e a avaliação integrativa dos resultados obtidos ${ }^{12}$.

Neste estudo, optou-se por privilegiar periódicos de divulgação científica, já que a revisão integrativa trabalha com evidências. Foi consultado o PubMed, por meio do software EndNote X7, e a Biblioteca Virtual em Saúde (BVS), utilizando-se as bases de dados SciELO (Scientific Electronic Library Online), Medline (Medical Literature Analysis and Retrieval System Online) e a Lilacs (Literatura Latino-Americana e do Caribe em Ciências da Saúde).

$\mathrm{Na}$ busca eletrônica dos artigos científicos e indexados nas bases de dados, utilizamos os seguintes Descritores das Ciências da Saúde (DeCS) e suas combinações nas línguas portuguesa, inglesa e espanhola: Estudantes de medicina ( $\mathrm{Me}$ dical students - Estudantes de Medicina), Drogas (Drugs - Drogas) e Prevalência (Prevalence - Prevalência).

Utilizamos os seguintes critérios de inclusão para selecionar os estudos: indexação de estudos nas respectivas bases de dados; relação direta com os descritores; idiomas de publicação português, inglês e espanhol; período de publicação do artigo compreendido entre janeiro de 2008 e dezembro de 2013, e estudos com pesquisas de campo, excluindo,assim, revisões de literatura. A coleta dos dados foi realizada seguindo os critérios de inclusão por dois pesquisadores de forma independente e, posteriormente, os resultados foram confrontados.

Ao associarmos os descritores estudantes de medicina, drogas e prevalência,encontramos 239 referências na Biblioteca Virtual de Saúde (BVS), sendo 94 publicações de 2008 a 2013. O total de 356 referências foi encontrado no PubMed 
pelo EndNote X7, sendo 135 artigos publicados no recorte de tempo citado.

Na primeira fase, realizou-se a leitura dos títulos dos artigos e seus resumos. Após essa análise, foram selecionados 30 artigos na BVS e 19 no PubMed. No confronto dos resultados das buscas entre as bases de dados, do total de 49 artigos, 14 se repetiram, restando, assim, 35 publicações, selecionadas para leitura integral dos textos. Na segunda fase, foi feita a leitura completa dos artigos, sendo excluídas as publicações que, embora contemplassem os descritores, não tratavam diretamente do tema objeto de estudo desta pesquisa. Após essa fase, foram selecionados 28 artigos, que compõem a amostra final para a presente revisão.

Na terceira e última fase, os 28 artigos foram analisados a partir da identificação de variáveis de interesse e conceitos-chave, conforme proposto em literatura específica acerca de revisão integrativa de literatura ${ }^{12}$. Empreendeu-se também uma análise de conteúdo, realizada de forma descritiva, qualitativa e integrativa, decompondo-se, assim, a comunicação textual em categorias analíticas ${ }^{13}$.

Dessa forma, procedeu-se à categorização dos dados extraídos dos estudos selecionados em grupos temáticos, o que possibilitou reunir o conhecimento produzido sobre o tema explorado na revisão. As categorias foram construídas de forma dedutiva, com base em critérios preestabelecidos que visam alcançar os objetivos propostos.

\section{RESULTADOS E DISCUSSÃO}

Por meio da análise dos 28 artigos, verificou-se que todos os estudos se enquadram na área da Saúde. A área com o maior número de publicações foi a Médica, com 24 artigos (85,71\%), seguida de Enfermagem, com quatro artigos (14,29\%). Não houve publicações de outras áreas do conhecimento. Esse dado indica que os maiores interessados na temática são da Medicina e que há uma escassez de trabalhos que compromete enxergar o problema por outras perspectivas.

Em relação ao ano das publicações, observou-se que 2008 foi o período com o maior número de artigos científicos publicados e indexados sobre a temática investigada, $11(39,2 \%)$, seguido dos anos 2009, com6 (21,4\%), 2010, também com 6 (21,4\%), 2011 e 2013, com 2 (7,14\%) artigos cada e 2012 com apenas $1(0,28 \%)$ estudo. Pode-se inferir que houve um decréscimo de publicações a partir de 2009, um dado preocupante, já que a análise dos estudos científicos evidencia um constante aumento da prevalência do uso de drogas entre estudantes de Medicina no decorrer dos anos.

Quanto aos periódicos, destacaram-se importantes revistas nacionais, dentre as quais merecem evidência a $R e$ - vista Brasileira de Educação Médica e o Jornal Brasileiro de Psiquiatra, com $2(7,14 \%)$ artigos cada. O periódico com o maior número de publicações foi a Revista Latino-Americana de Enfermagem, com 4 (14,2\%). O Brasil foi o país com o maior número de estudos, com10 (35,7\%), seguido dos Estados Unidos e Nepal, com três artigos cada. Outros países também com estudos foram Colômbia, Alemanha, Macedônia, Índia, Nicarágua, Chile, Peru, Etiópia, Honduras, Líbano, Hungria e Paquistão.

Nesse sentido, o método de análise da temática possibilitou categorizar, interpretar e agrupar dados. Desse agrupamento emergiram três categorias temáticas: (1) prevalência do uso de drogas entre os estudantes de Medicina (categoria dividida em drogas lícitas e ilícitas); (2) fatores que propiciam o uso de drogas entre os estudantes de Medicina;(3) análise referente aos estudantes brasileiros de Medicina.

\section{Prevalência do uso de drogas lícitas entre os estudantes de Medicina}

Grande parte dos autores detectou, em suas pesquisas, elevadas taxas de consumo de drogas lícitas entre os estudantes de Medicina, com prevalência doálcool 5,7,14-19,22,23,25,29-31,33,34. Comparando os estudantes de Medicina com outros estudantes universitários, pesquisas ${ }^{25,37}$ afirmam que aqueles utilizam mais bebida alcoólica do que estudantes de outros cursos. Porém, pesquisas de alguns autores ${ }^{30-32}$ apontam que não se pode afirmar que exista uma diferença estatisticamente significativa entre as duas populações estudadas.

Segundo Paduani et al. ${ }^{7}$, a ingestão de bebidas alcoólicas aumenta no decorrer dos períodos do curso de Medicina. Levanta-se, assim, a hipótese de que algumas características do curso médico contribuem para esse aumento no consumo, já que a formação médica impõe maiores cargas emocionais ao aluno, como um contato mais direto com os pacientes e rigores no estágio ${ }^{31}$.Observou-se que alunos no primeiro e segundo anos ingerem mais bebida alcoólica do que os do terceiro e quarto anos ${ }^{17}$. Em contrapartida a este dado, alguns estudos não determinaram uma variação significativa na intensidade do uso em relação aos diferentes períodos do curso de Medicina ${ }^{15,17}$.

Ao se avaliar a prevalência comparativa do consumo de drogas lícitas entre médicos e estudantes de Medicina ${ }^{15}$, notou-se que o uso de bebidas alcoólicas é maior pelos estudantes do que pelos médicos. Apesar da existência desse dado no estudo citado, uma porcentagem significativa tanto de estudantes como de médicos relatou ingestões mais elevadas do que a quantidade diária recomendada. Além disso, constatou-se que médicos e estudantes ingerem quantidades perigosas 
de álcool, sendo médicos do sexo masculino em maior número e estudantes femininas em maior número ${ }^{15}$.

A segunda droga lícita prevalente é o tabaco ${ }^{5,14,16,17-20,23-26}$, porém, ao contrário do álcool, a prevalência do tabagismo parece atingir níveis baixos entre os estudantes ${ }^{7,18}$. Nota-se que, por algum motivo, eles têm mais consciência dos malefícios do cigarro em longo prazo. Tal condição é considerada de alta relevância, já que a classe médica tem importância fundamental no aconselhamento dos pacientes em relação ao abandono ou à não iniciação ao uso do tabaco? .

Há evidências de que existe relação entre o consumo de álcool e de outras drogas entorpecentes, além de tabagismo como sendo o principal, corroborando a hipótese de que o alcoolista tem predisposição para outros comportamentos de risco ${ }^{17,21,29,36}$. Embora o tabaco seja a segunda substância mais consumida, alguns estudos demonstraram que não está havendo um uso frequente dessa droga ${ }^{17,18}$.Supõe-se que as campanhas contra o tabagismo nos últimos cinco anos podem ser responsáveis por essa mudança de conduta ${ }^{7,17}$

As publicações científicas analisadas evidenciaram em suas pesquisas que os estudantes de Medicina apresentam vários comportamentos de risco associados ao consumo de drogas lícitas. Dentre eles, considerou-se a amnésia alcoólica, o não uso de preservativo, maior número de parceiros sexuais, dependência de jogos de azar, internet, envolvimento em acidentes de trânsito, brigas e oniomania (compulsão por fazer

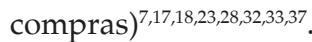

Portanto, mesmo conhecendo os danos potenciais e diretos que essas substâncias, mesmo lícitas, podem causar ao próprio organismo e à sociedade em geral, os estudantes de Medicina ainda buscam alguma utilidade no uso dessas drogas, porém estão sujeitos e vulneráveis às consequências desse consumo.

\section{Prevalência do uso de drogas ilícitas entre os estudantes de Medicina}

Voigt et al. ${ }^{15}$ constataram que, em relação às drogas ilícitas, a maioria (dois terços) dos estudantes de Medicina pesquisados negou qualquer consumo. Dentre aqueles que relataram consumir esse tipo de droga, a prevalência se dá muito mais em homens do que em mulheres. O mesmo foi descrito por Santos et al..$^{23}$, que também encontraram uma predisposição masculina para o uso de substâncias consideradas ilícitas. Já na pesquisa de Vásquez et al. ${ }^{14}$, foi constatado que o consumo entre as mulheres está se aproximando ao dos homens, principalmente em relação à maconha.

A maconha se apresenta como a primeira droga ilícita mais utilizada pelos estudantes de Medicina ${ }^{5,17,20,22-25,33} \mathrm{e}$ tam- bém a prevalente entre homens ${ }^{18}$.É considerada a substância ilícita de mais fácil entrada no ambiente universitário e de maior uso entre estudantes em geral e de Medicina. Uma das possíveis explicações desse fato é a percepção de que a maconha é uma "droga leve", sem muitas consequências para a saúde do indivíduo, em contraste com outras drogas lícitas ${ }^{23}$.

No estudo realizado por Budhathoki et al.27, a utilização da maconha esteve em primeiro lugar entre os estudantes de Medicina do Nepal que cursavam o terceiro ano, diferenciando-se, assim, de outros estudos em que o álcool se apresentou como a principal droga de uso. Ainda nesse estudo, mais da metade dos entrevistados envolvidos em algum tipo de substância afirmou que o uso começou após se juntarem à Faculdade de Medicina.

Quanto à utilização da cocaína, esta apresenta prevalência acima da média da população brasileira. Porém, quando comparada a outras drogas, é a que menos se consome entre os estudantes de Medicina ${ }^{16,35}$. No estudo de Romero et al.$^{26}$, a prevalência anual do uso de cocaína foi bastante inferior quando comparada à prevalência em estudantes universitários chilenos, entre jovens chilenos e até mesmo na população chilena em geral. Diante disso, acredita-se que os estudantes de Medicina podem ser mais conscientes dos danos e dos riscos de dependência dessas drogas, que atuam como um elemento de dissuasão, o que pode explicar seu baixo consumo ${ }^{26}$.

No estudo de Pineda-Rodriguez ${ }^{28}$, realizado na Faculdade de Medicina San Luis Gonzaga de Icano,no Peru,comum a amostra de 195 estudantes pesquisados, quando perguntados sobre a dificuldade de obter drogas ilícitas, $67,18 \%$ responderam ser indiferentes ou não saber, $19,49 \%$ responderam muito difícil ou difícil e $12 \%$ fácil ou muito fácil.Diante dessa perspectiva de obter a droga ilícita,Oliveira Junior et al. ${ }^{16}$ afirmaram que existe uma relação entre consumo de drogas e acesso fácil às substâncias por meio de traficantes que as vendem nos arredores das universidades. Entretanto, não existem estatísticas significativas sobre essa relação entre universitários e traficantes próximos às universidades, sendo necessários estudos mais aprofundados a respeito.

Foi observada também alta prevalência de consumo de benzodiazepínicos sem receita médica pelos estudantes de Medicina. Nesse estudo, o uso de antidepressivos sem prescrição foi maior nas mulheres ${ }^{26}$. Outros estudos também apontaram que as mulheres consomem não só mais antidepressivos como também ansiolíticos, mas as publicações não relatam se existe prescrição médica ou não e, assim,não se pode afirmar que se trate de uso ilícito. Necessita-se, portanto, de mais estudos sobre esta questão $0^{18,26,35}$. 


\section{Fatores que propiciam o uso de drogas entre os estudantes} de Medicina

Alguns estudos mostraram que o consumo de álcool e outras substâncias lícitas e ilícitas está bastante presente na comunidade médica ${ }^{7,14-17}$ e que frequentemente se inicia durante os anos de faculdade $\mathrm{e}^{7,14,16,17,18}$. Com isso, vários autores $7,14-22,34$ se preocuparam em discorrer sobre os fatores que propiciam o uso de drogas entre os estudantes de Medicina.

Segundo Silveira et al. ${ }^{31}$, a prevalência do uso de drogas tende a aumentar a partir do primeiro até o sexto ano letivo em escolas de Medicina para a maioria das substâncias, o que leva à hipótese de que algumas características do curso médico podem contribuir para esse aumento no consumo. Shah et al. ${ }^{4}$ afirmaram que o ano de escolaridade médica está associado a taxas mais elevadas de consumo devido a uma mudança na fonte de ansiedade, como o fato de os alunos se deslocarem da fase pré-clínica para períodos de clínica, onde se deparam com diferentes níveis de pressão acadêmica, carga de trabalho e aumento na frequência da síndrome de burnout. Oliveira Júnior et al. ${ }^{16}$ identificaram que o ano letivo tem influenciado não só o consumo de drogas, mas também a diminuição, na prática clínica, de aconselhamento do paciente sobre os efeitos e as consequências do uso de álcool e outras drogas.

Fatores estressantes ou desencadeantes - como a pressão a que o estudante de Medicina está submetido, carga horária excessiva com grande quantidade e responsabilidade de trabalho, privação do convívio familiar e lazer, o fato de lidar com a vida, o sofrimento humano e a morte ${ }^{7}$, cansaço $\mathrm{O}^{14,16,17}$, tentativa de melhorar o rendimento acadêmico ${ }^{16}$, aliviar as tensões ${ }^{17,18,22}$, bem como o relato de maus-tratos dentro da escola médica ${ }^{38}$ - possivelmente contribuem para a gênese dessa prevalência de uso de drogas. Alguns estudos ${ }^{19,20,21,34}$ têm proposto que altos níveis de ansiedade e estresse durante a escolaridade médica podem levar ao abuso de substâncias, incluindo o consumo excessivo de álcool, sendo reconhecido como um dos métodos comuns de redução de estresse entre os estudantes de Medicina.

Entre as situações propícias ao consumo pelos estudantes estão as festas da faculdade, que foi a opção prevalente $(72,45 \%)$, seguida de pós-provas da faculdade $(11,74 \%)$. A alternativa "ao final de um dia estressante de faculdade" foi escolhida por 3,57\% dos alunos, e $21,24 \%$ deles optaram por todas as situações citadas ${ }^{7}$.

Os estudos, então, mostraram que a educação médica e a profissão médica estão entre as mais exigentes e estressantes no que tange a sua formação. Evidenciam-se altas taxas de depressão, ansiedade e outros sintomas psiquiátricos que influenciam os estilos de vida e as atitudes dessa população. Muitas vezes, as substâncias psicoativas lícitas ou ilícitas são a única "fuga" encontrada por esses estudantes em tal situação.

\section{Análise referente aos estudantes brasileiros de Medicina}

Dos artigos científicos publicados e indexados cujas pesquisas se referem ao estudante de Medicina do Brasil, oito estudos são de periódicos brasileiros e dois foram publicados nos Estados Unidos, no International Journal of Adolescent Medicine Health e na Addictive Behaviors.Assim,em relação às pesquisas brasileiras,houve escassez de trabalhos entre 2010 e 2013, sendo um artigo publicado em 2010 e outro em 2013. O ano com mais publicações foi 2008,com cinco artigos,e em seguida o de 2009, com três trabalhos.

A maioria dos trabalhos concluiu que há prevalência alta do uso de drogas entre os universitários brasileiros, com atenção especial aos do curso de Medicina. $\mathrm{O}$ álcool, assim como no contexto mundial, também foi identificado como a principal substância de uso, permanecendo em segundo o tabaco e em seguida a droga ilícita, a maconha ${ }^{5,7,16,18,22,23,25,33}$.

A adesão às drogas foi mais encontrada em estudantes solteiros, do sexo masculino. Porém, houve evidências de que o consumo de álcool quanto à diferenciação de gênero no Brasil é quase inexistente, podendo-se concluir que as mulheres estão em potencial uso e constante aumento de suaprevalência ${ }^{5,7,18,31}$.

No estudo de Pereira et al. ${ }^{18}$, observou-se que as bebidas preferidas pelos estudantes foram a cerveja em primeiro lugar e a vodca em segundo. Os autores desse estudo ressaltaram que, apesar de a vodca figurar entre as bebidas mais consumidas, as condições nas quais ela é ingerida, ou seja, sob a forma de coquetéis ou drinques, está muito presente nas festas organizadas por tais universitários. Nesse sentido, Paduani et al. ${ }^{7}$ detectaram que as festas de faculdade foram consideradas a situação mais propícia ao consumo, acrescentando ainda a utilização no final de um dia estressante e no período após as provas.

Com relação ao uso do tabaco, as pesquisas brasileiras demonstraram que não há uso frequente e em demasia entre os estudantes de Medicina 7,17,18. Existe, sim, nos estudos, a prevalência do tabaco como a segunda substância mais utilizada, porém apresentando taxas baixas de consumo. Além disso, se faz presente a hipótese de que quem utiliza o álcool tem maior predisposição ao uso de outras substâncias, não necessariamente o tabaco ${ }^{16,17}$.

Quanto às drogas ilícitas, no Brasil a maconha também é a terceira substância mais usada entre os estudantes de Medicina, sendo os do sexo masculino os usuários de maior prevalência comparados às estudantes do sexo feminino ${ }^{23}$. Pereira $e t$ al. ${ }^{18}$ apontaram em seu estudo que a idade de experimentação 
de drogas ilícitas foi de 16 a 18 anos. Diante desse dado, supõe-se que a idade de experimentação esteja relacionada com vários aspectos que envolvem o ambiente universitário e pré-universitário, tais como morar sozinho, em casa de parentes ou em repúblicas, aquisição de novas amizades, aceitação social, festas universitárias, maior sensação de liberdade e autonomia.

Oliveira et al..$^{25}$ detectaram uma mudança acentuada no perfil de uso de drogas entre estudantes de Medicina. $\mathrm{O}$ uso de inalantes subiu,tendo atingido a posição de segunda droga mais consumida, ultrapassando a maconha e até mesmo o tabaco. Além disso, essa droga foi prevalente entre os alunos nos primeiros anos escolares, enquanto o uso recreativo de anticolinérgicos e ecstasy era mais comum entre os alunos nos anos posteriores.

Petroianu et al. ${ }^{17}$ mostraram que a ingestão de bebidas alcoólicas está diretamente relacionada com o uso de outras substâncias psicoativas, incluindo as ilícitas, corroborando a hipótese de que o alcoolista tem predisposição para outros comportamentos de risco.O uso de maconha ou haxixe foi relatado por boa parte desses estudantes, e houve evidência de que aqueles que trabalhavam consumiam menos esse tipo de substância.

Gonçalves e Silvany Neto $^{22}$, ao avaliarem a dimensão psicológica da qualidade de vida dos estudantes de Medicina da Universidade Federal da Bahia, afirmaram que ouso passado ou atual de drogas ilícitas mostrou-se fator de aumento de prevalência de baixa dimensão psicológica da qualidade de vida(DPQV) entre esses estudantes. A busca por drogas pode ser impulsionada pelo enfrentamento de problemas, sendo consequência de baixa DPQV, e também pode contribuir como causa de baixa qualidade de vida devido aos efeitos diretos das próprias substâncias usadas e à potencial preocupação com o caráter ilícito.

\section{CONCLUSÕES}

Várias características do curso de Medicina o tornam potencialmente atuante na qualidade de vida dos estudantes. Diversos autores identificaram fatores estressantes na educação médica que levam a consequências na saúde desses alunos, como o uso e/ou abuso de drogas lícitas e ilícitas.

O consumo dessas substâncias é uma questão de saúde pública, e a análise da sua prevalência, do seu padrão de consumo e dos fatores desencadeantes, principalmente quando presentes entre estudantes da área médica, se torna importante. $\mathrm{O}$ conhecimento dessas condições e de suas consequências poderá orientar ações que visem modificar os fatores relacionados ao curso que interferem negativamente na saúde desses estudantes, visto que merecem um enfoque diferenciado em relação a essas atitudes. É importante lembrar que eles serão os promotores de saúde junto à comunidade.

A detecção do uso e abuso de álcool e outras drogas entre os acadêmicos de Medicina é relevante, uma vez que estes estudantes, futuros profissionais da saúde, serão responsáveis pelo diagnóstico, pelas intervenções e pelos encaminhamentos de pacientes com as mais diversas doenças, inclusive as relacionadas ao uso e abuso de drogas. Tal prática pode interferir na habilidade desses profissionais para tratar dessa problemática, com possíveis impactos na profissão e impactos sociais, já que se pressupõe que o médico servirá de modelo para seus pacientes e outros profissionais de saúde que com ele convivem.

A existência de um número considerável e preocupante de estudantes que fazem uso destas drogas lícitas e ilícitas merece a atenção dos representantes das universidades para que se implementem políticas de controle e redução de uso de drogas no âmbito universitário. Algumas pesquisas concluíram que estratégias de prevenção são necessárias ${ }^{7,14,16,17,18,24,25}$ e apontaram a importância da instituição educacional na prevenção da dependência química ${ }^{14,16,17,18,24,25}$. Além disso, sugerem a realização de campanhas que alertem sobre os malefícios do consumo excessivo, já que foram demonstradas ocasiões em que os estudantes apresentaram comportamentos de risco, inclusive amnésia alcoólica.

Este estudo constatou evidências de um grave problema em curso nas Faculdades de Medicina, que é a grande e constante prevalência de uso de drogas lícitas ou ilícitas entre os estudantes e fatores intrínsecos ao curso que podem desencadear o início ou a continuidade dessa prática. Os achados deste trabalho indicam que informações sobre álcool e outras drogas devem ser prioridade em escolas de Medicina, as quais devem promover programas preventivos direcionados a essa população.

\section{REFERÊNCIAS}

1. Santos JAT, Oliveira MLF. Políticas públicas sobre álcool e outras drogas: breve resgate histórico.J NursHealth2012;1(2):82-93.

2. Organização Mundial da Saúde. Terminology \& Classification. [capturado em 12 mar. 2014]; Disponível em: http:/ / www.who.int/substance_abuse/terminology/psychoactive_substances/en

3. Carlini EA, Galduróz JC, Silva AAB, Noto AR, Fonseca AM, Carlini CM,et al. II Levantamento domiciliar sobre o uso de drogas psicotrópicas no Brasil: estudo envolvendo as 108 maiores cidades do país. São Paulo: CEBRID, UNIFESP, 2006. 
4. Shah AA, Bazargan-Hejazi S, Lindstrom RW, Wolf KE. Prevalence of At-Risk Drinking among a National Sample of Medical Students. Subst Abus. 2009; 30(2): 141-149.

5. Tockus D,Gonçalves PS. Detecção do uso de drogas de abuso por estudantes de medicina de uma universidade privada. J Bras Psiquiatr. 2008;57(3):184-187.

6. Andrade AG, Duarte PCAV, Oliveira LG. I Levantamento Nacional sobre o Uso de Álcool, Tabaco e Outras Drogas entre Universitários das 27 Capitais Brasileiras. Brasília: SENAD, 2010. [capturado em 20 set. 2013]. Disponível em: http:/ / obid.senad.gov.br/portais/OBID/biblio teca/documentos/Publicacoes/Universitarios_2010/328160.pdf

7. Paduani GF, Barbosa GA, Morais JCR, Pereira JCP, Almeida MF, Prado MM, et al. Consumo de álcool e fumo entre os estudantes da Faculdade de Medicina da Universidade Federal de Uberlândia. Rev bras educ med. 2008;32(1).

8. Pedrosa AAS. Perfil epidemiológico do consumo de álcool e fatores relacionados em estudantes universitários das ciências da saúde de Maceió/Alagoas. Rio de Janeiro; 2009. Mestrado [Dissertação] - Escola Nacional de Saúde Pública Sergio Arouca.

9. Roberto A, Almeida A. Saúde mental de estudantes de medicina, Acta Med Port. 2011; 24 (S2): 279-286.

10. Guimarães KBS. Estresse e a formação médica: implicações na saúde mental dos estudantes. São Paulo; 2005. Mestrado [Dissertação] - Faculdade de Ciências e Letras de Assis - Universidade Estadual Paulista.

11. Gallassi AD, Malbergier A, Andrade AG. Cuidados no atendimento de estudantes que usam substância. In: Baldassin S, org.Atendimento psicológico aos estudantes de medicina: técnica e ética. São Paulo: Edipro, 2012. p. 93100.

12. Mendes KDS, Silveira RCCP, Galvão CM. Revisão Integrativa: método de pesquisa para incorporação de evidências na saúde e na enfermagem. Texto Contexto-Enferm. 2008, 17(4):758-64.

13. ChizzottiA. Pesquisa qualitativa em ciências humanas e sociais. Petrópolis: Vozes; 2006.

14. Vásquez EMM, Cunningham J, Brands B, Strike C, Wright MGM. Consumo percibido y uso de drogas lícitas e ilícitas em Estudiantes universitários em La ciudad de Medellín, Colombia. Rev Latino-am Enfermagem 2009; 17(Esp.):88692.

15. Voigt K, Twork S, Mittag D, Gobel A, Voigt R, Klewer J,et al. Consumption of alcohol, cigarettes and illegal substances among physicians and medical students in Brandenburg and Saxony (Germany).BMC Health Services Research, 2009, 9:219.
16. Oliveira Junior HP, Brands B, Cunningham J, Strike C, Wright MGM. Percepção dos estudantes universitários sobre o consumo de drogas entre seus pares no ABC Paulista, São Paulo, Brasil. Rev Latino-am Enfermagem 2009; 17(Esp.):871-7.

17. Petroianu A, Reis DCF, Cunha BDS, Souza DM. Prevalência do consumo de álcool, tabaco e entorpecentes por estudantes de medicina da universidade federal de Minas Gerais. Rev Assoc Med Bras 2010; 56(5): 568-7.

18. Pereira DS, Souza RS, Buaiz V, Siqueira MM. Uso de substâncias psicoativas entre universitários de medicina da universidade federal do Espírito Santo. J Bras Psiquiatr. 2008; 57(3):188-195.

19. Mancevska S, Bozinovska L, Tecce J, Pluncevik-Gligoroska J, Sivevska-Smilevska E. Depression, anxiety and substance use in medical students in the Republic of Macedonia. Bratisl Lek Listy. 2008;109(12):568-72.

20. Khanal P, Ghimire RH, Gautam B, Dhungana SK, Parajuli $\mathrm{P}$, Jaiswal AK, et al. Substance use among medical students in Kathmandu Valley. JNMA J Nepal Med Assoc. 2010;50(180):267-72.

21. Rai D, Gaete J, Girotra S, Pal HR, Araya R.Substance use among medical students: time to reignite the debate? The National Medical Journal of India 2008;21(2):75-8.

22. Gonçalves SS, Silvany Neto AM. Dimensão psicológica da qualidade de vida de estudantes de medicina. Revista brasileira de educação médica. 2013; 37 (3): 385-395.

23. Santos WJ,SakaeTM, Escobar BT. Relação entre o uso de drogas e comportamento sexual de risco em universitários de um curso de medicina. Revista da AMRIGS 2009; 53 (2): 156-164.

24. Castro N, Cortés P, Vasters GP, Costa Jr ML.Uso de drogas entre estudiantes de La Facultad de Ciencias Médicas de La Universidad Nacional Autónoma de Nicaragua, León, Nicaragua, Rev. Latino-Am. Enfermagem 2010; 18(Spec):606-12.

25. Oliveira LG, Barroso LP, Wagner GA, Ponce JC, Malbergier A, Stempliuk VA, et al. Drugconsumption among medical students in São Paulo, Brazil: influences of gender and academic year. Rev. Bras. Psiquiatr. 2009;31(3):227-239.

26. Romero MI, Santander J, Hitschfeld MJ, Labbé M, Zamora V. Consumo de sustancias ilícitas y psicotrópicos entre los estudiantes de medicina de La Pontificia Universidad Católica de Chile.Rev Med Chil 2009; 137(4): 459-465.

27. Budhathoki N, Shrestha MK, Acharya N, Manandhar A. Substance use among third year medical students of Nepal. J Nepal Health Res Counc 2010;8(16):15-8. 
28. Pineda-Rodriguez AA. Percepción sobre el consumo de sustâncias psicoactivas por estudiantes de medicina de La provincia de Ica, Perú 2010.Rev. Méd.Panacea 2011; 1(2): 44-46.

29. Deressa W, Azazh A. Substance use and its predictors among undergraduate medical students of Addis Ababa University in Ethiopia. BMC Public Health 2011;11:660.

30. Buchanan JC, Pillon SC. O uso de drogas entre estudantes de medicina em Tegucigalpa, Honduras. Rev Latino-Am Enfermagem 2008; 16(n.esp): 595-600.

31. Silveira DX, Rosa-Oliveira R, Di Pietro M, Niel M, Doering-Silveira E, Jorge MR. Evolutional pattern of drug use by medical students. Addictive Behaviors 2008; 33 :490-495.

32. Moaouad J, Kazour F, Haddad R, Rouhayem J, Chammai R, Richa S. Chemical and behavioural addiction of medical students: comparative study in Lebanese students. L'Encéphale2012; 38(6):467-72.

33. Carvalho KA, Sant'Anna MJ, Coates V, Omar HA. Medical students: abuse of psychoactive substances and sexuality aspects. Int J Adolesc Med Health.2008; 20(3):321-8.

34. Shekhar S. Substance abuse among medical students and doctors: a call for action.Natl Med J India 2008; 21(2):57-9.

35. Horowitz A, Galanter M, Dermatis H, Franklin J.Use of and attitudes toward club drugs by medical students.J Addict Dis. 2008;27(4):35-42.

36. Ghimire A, Sharma B, Niraula SR, Devkota S, Pradhan PM. Smoking habit among male medical and dental students of B.P.Koirala Institute of Health Sciences, Nepal. Kathmandu UnivMed J (KUMJ) 2013; 11(41):32-6.
37. Bíró E, Balajti I, Adány R, Kósa K. Survey of the state of health and health behavior among medical students in Hungary. Orv Hetil. 2008; 16;149(46):2165-71.

38. Shoukat S, Anis M, Kella DK, Qazi F, Samad F, Mir F, et al. Prevalence of Mistreatment or Belittlement among Medical Students - A Cross Sectional Survey at a Private Medical School in Karachi, Pakistan. PLoS ONE 2010; 5(10): e13429.

\section{CONTRIBUIÇÃO DOS AUTORES}

Cleomara de Souza Machado: Contribuiu na concepção do artigo, pesquisa bibliográfica, coleta de dados, análise e interpretação dos dados e na redação final do texto. Talles Mendes de Moura: Contribuiu na concepção do artigo, pesquisa bibliográfica, coleta de dados, análise e interpretação dos dados e na redação final do texto.Rogério José de Almeida: Contribuiu na concepção do artigo, coleta de dados, análise e interpretação dos dados, redação final do texto, orientador do trabalho.

\section{CONFLITO DE INTERESSES}

Declarou não haver.

\section{ENDEREÇO PARA CORRESPONDÊNCIA}

Cleomara de Souza Machado

Rua Abaeté - Qd. 101 - chácara 14

Jardim Guanabara I - Goiânia

CEP 74675-010 - GO

E-mail:csmcleo@hotmail.com 